\title{
Dr. Luis Jasso Gutiérrez
}

\author{
Onofre Muñoz-Hernández y Fortino Solórzano-Santos ${ }^{2 *}$ \\ ${ }^{1}$ Comisión Nacional de Arbitraje Médico; ${ }^{2}$ Hospital Infantil de México Federico Gómez. Ciudad de México, México
}

El Dr. Luis Jasso Gutiérrez nació en Tenosique, Tabasco, lugar del que siempre se sintió orgulloso, y reflejó sus vivencias en esa población en su libro Ecos del Pasado.

Estudió la carrera de Medicina en la Facultad de Medicina de la Universidad Nacional Autónoma de México (UNAM). Su inquietud académica le llevó a realizar la especialidad en Pediatría Médica en el Hospital de Pediatría del Centro Médico Nacional del Instituto Mexicano del Seguro Social (IMSS), con reconocimiento por la UNAM, y posteriormente una segunda especialidad en Neonatología por la UNAM y la Maestría en Ciencias Químicas (área de Bioquímica). Su carrera laboral la desarrolló en el Hospital de Pediatría del Centro Médico Nacional. Al incorporarse como médico adscrito al Servicio de Neonatología, fue becado en su primer año para visitar diferentes centros de neonatología en los Estados Unidos de América.

Años después fue designado Jefe del Servicio, donde desarrolló un área de neonatología que fue modelo y escuela de numerosos neonatólogos connotados.

Como parte de su aportación a la Neonatología elaboró el Manual de Neonatología Práctica, texto que fue esencial en la formación de pediatras y neonatólogos. En este periodo, la energía y la disciplina fueron características que imprimió a todos aquellos que tuvieron la oportunidad de hacer alguna estancia en su servicio; las tradicionales entregas de guardia eran momentos de gran estrés y aprendizaje para los residentes en formación. Después fue nombrado Jefe de Estudios de Posgrado en el IMSS. El sismo de 1985 provocó el cierre y la demolición del Hospital de Pediatría. Sin embargo, en 1991 se abrió el nuevo Hospital de Pediatría del Centro Médico Nacional Siglo XXI, donde se reincorporó como médico tratante, puesto que ejerció varios meses y sustituyó al Dr. Onofre Muñoz en la dirección del hospital. Otro cargo que ocupó fue el de Presidente de la Comisión de Cuadros Básicos en el IMSS. En el año 2007 fue nombrado Jefe del Departamento de Evaluación y Análisis de Medicamentos de la Dirección de Investigación del Hospital Infantil de México Federico Gómez y Presidente del Comité de Ética en Investigación (Figura 1).

Dentro de la docencia destaca también el haber actuado como Profesor de Asignatura y Tutor Académico de Maestrías y Doctorados en la Facultad de Medicina de la UNAM. Su natural interés le permitió publicar muchos trabajos de investigación, en los cuales plasmó el análisis de la experiencia vivida en el servicio de neonatología y en las áreas donde laboró (aspectos de educación, farmacovigilancia y ética, entre otros). Publicó también un libro sobre el niño con síndrome de Down, en el que vertió su interés por los niños con capacidades diferentes (Figura 2). Fue invitado a contribuir con varios capítulos en libros de otros destacados neonatólogos.

\section{Correspondencia:}

*Fortino Solórzano-Santos

E-mail: solorzanof056@gmail.com

1665-1146/@ 2021 Hospital Infantil de México Federico Gómez. Publicado por Permanyer. Este es un artículo open access bajo la licencia CC BY-NC-ND (http://creativecommons.org/licenses/by-nc-nd/4.0/).
Disponible en internet: 17-09-2021 Bol Med Hosp Infant Mex. 2021;78(5):497-499

www.bmhim.com 


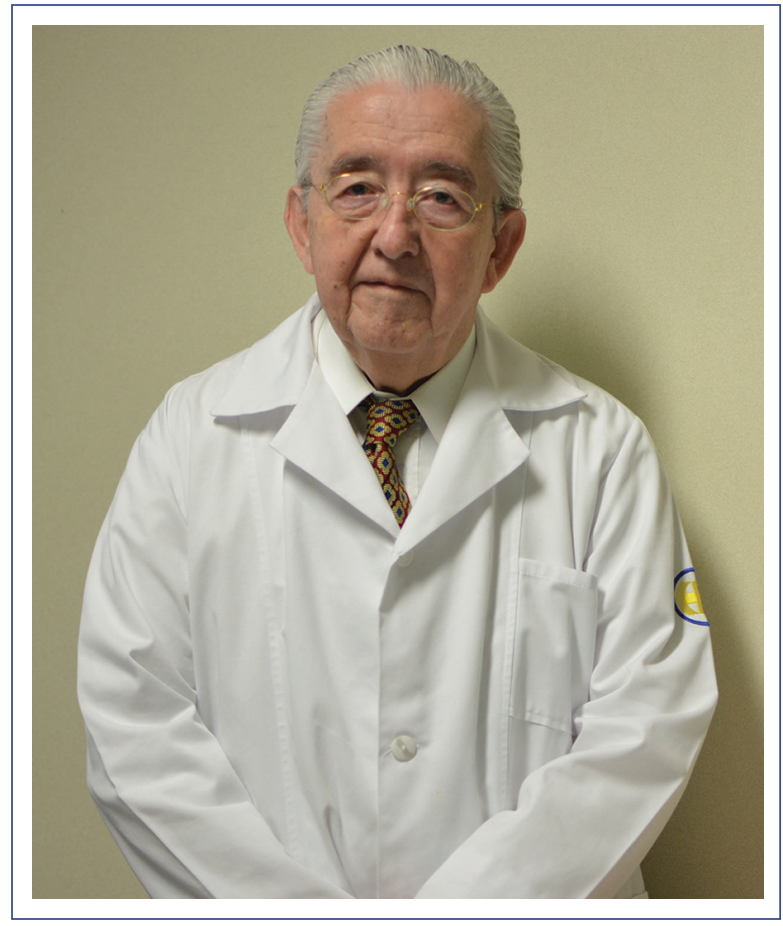

Figura 1. Fungió como Jefe del Departamento de Evaluación y Análisis de Medicamentos de la Dirección de Investigación del Hospital Infantil de México Federico Gómez desde 2007, donde también se desempeñó como Presidente del Comité de Ética en Investigación.

Su actividad laboral y académica fue reconocida, lo que le permitió ingresar a la Academia Nacional de Medicina y la Academia Mexicana de Pediatría, y ser miembro honorífico de varias sociedades de pediatría y neonatología tanto en México como en el extranjero (Figura 3).

El Dr. Luis Jasso fue un amante de la poesía, pasión que mantuvo desde su etapa de juventud hasta el final de sus días. De su paisano, Carlos Pellicer Cámara, poeta tabasqueño, es de quien más disfrutaba declamar sus poemas. La música fue otra de sus preferencias. Disfrutaba tocando la marimba y, sobre todo, le gustaba cantar en cada ocasión que se presentaba la oportunidad. Aunque era conocedor de diversos géneros, la música romántica era la que más le entusiasmaba.

El Dr. Luis Jasso siempre insistió en que el recién nacido era fisiológicamente muy diferente de otras edades pediátricas, que los avances tecnológicos harían que se enfrentara con más frecuencia a la atención de recién nacidos minúsculos y que habría que realizar todos los esfuerzos para reducir su mortalidad. Todo aquel que atendiera a un recién nacido tendría la

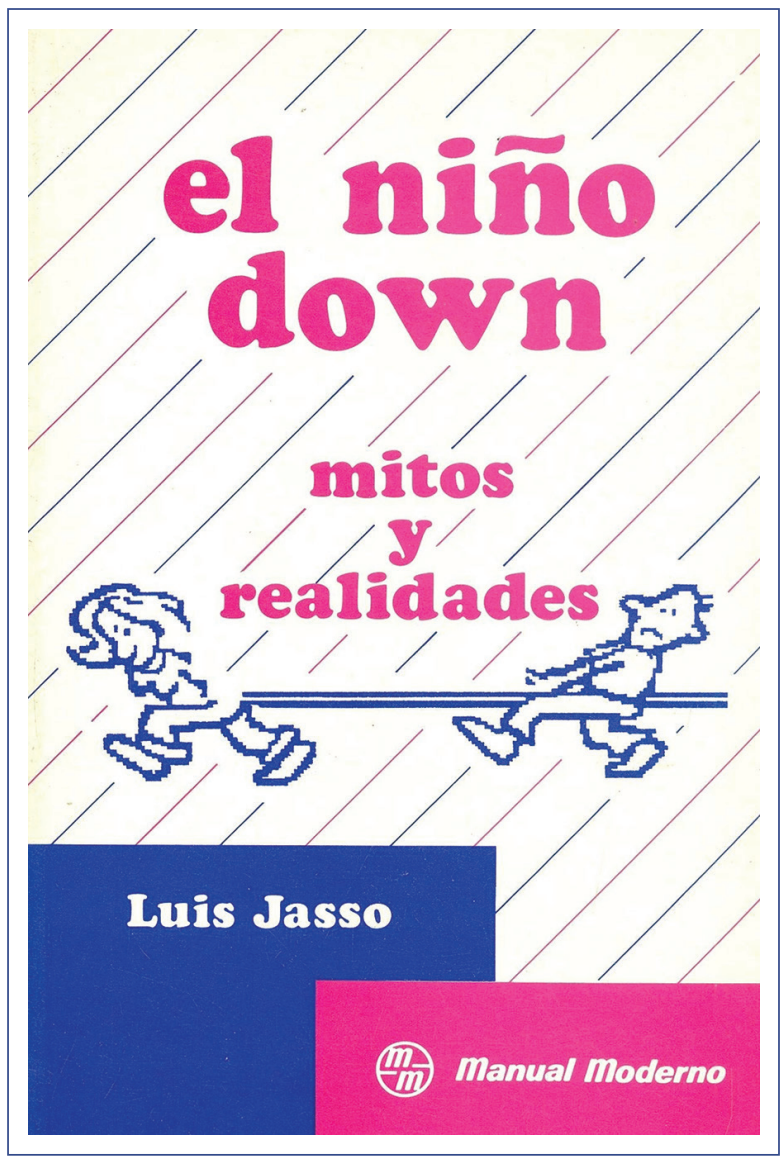

Figura 2. El niño Down, mitos y realidades es una obra única en la que el autor aborda los aspectos biológicos, médicos, psicológicos, educativos y sociales del niño con síndrome de Down de manera integral y con un enfoque humanista.

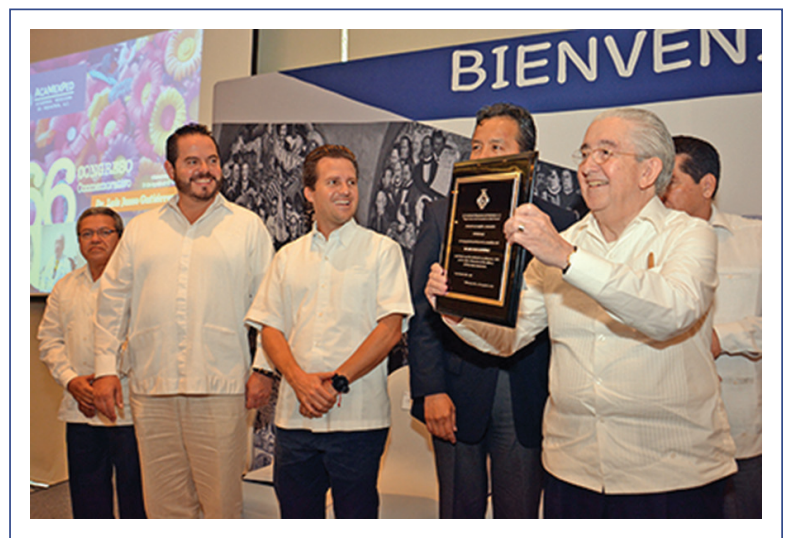

Figura 3. Reconocimiento al médico tabasqueño Luis Jasso Gutiérrez, maestro de la pediatría y la neonatología, por sus aportaciones a la pediatría y al Hospital del Niño Rodolfo Nieto Padrón. Villahermosa, Tabasco, México, 2017.

obligación de dominar la farmacología de los medicamentos que pretendiera prescribir y mantener una 
continua capacitación para estar a la par de los avances en el conocimiento.

Falleció en la Ciudad de México en el mes de marzo de 2021, causando una gran consternación entre los médicos pediatras, neonatólogos y de todas las especialidades que tuvieron la oportunidad de compartir con una persona tan distinguida. Seguramente muchas generaciones de pediatras y neonatólogos le tendrán presente de manera continua, cuando apliquen los conocimientos que él sembró a lo largo de los años.

En especial, el Dr. Onofre Muñoz y la Dra. Martha Morales han perdido un gran amigo y compañero, con el que transitaron juntos en el posgrado, en el ejercicio profesional de la pediatría, en la vida académica y en la convivencia familiar.

Descanse en paz, Dr. Luis Jasso Gutiérrez. 